\section{Beyond measurement: taking bold multisector actions towards zero catastrophic costs and suffering due to TB}

We agree with Timire et al. that all financial barriers faced by people with tuberculosis (TB) at all stages of the care cascade should be addressed if we are to achieve the ambitious targets set by the End TB Strategy. ${ }^{1}$ Further research into economic hardship during pathways to care, and also after treatment completion, is warranted. Several studies are ongoing, including 'TBSequel', which tracks costs after completed TB treatment in four African countries. ${ }^{2}$

Although national TB patient cost surveys focus on diagnosed TB patients, they also aim to capture retrospective information about pre-diagnosis costs related to seeking care. ${ }^{3}$ In addition, the survey instrument can be incorporated into patient pathway analyses, prospective studies and trials, and thereby, provide important complementary information.

The design and sampling strategy are as important as the choice of the instrument. Even studies with inception during the diagnostic pathway will inevitably miss those that do not seek or receive any care, or do so at facilities that are not captured in the study design. Prospectively collecting patient costs from symptomatic individuals as part of a TB prevalence survey is an attractive design, but challenging to implement, due for example, to the geographical spread of small numbers of untracked patients.

We welcome the suggestion by Timire et al. for more evaluations of different social protection interventions. Findings from ongoing or planned intervention studies (such as in Viet Nam, Nepal, Uganda and Mozambique), aiming to improve completion of the care pathway through different modalities of socio-economic support will contribute important evidence. Several of these studies are linked to the Social Protection Action Research \& Knowledge Sharing (SPARKS), ${ }^{4}$ an international interdisciplinary research network on social protection, aiming to facilitate networking and knowledge sharing between academia, public health practitioners, international organisations and civil society. Governmentled schemes such as the Direct Benefit Transfer in India may encourage people to engage with care, thus addressing another gap that is not tackled by looking at individuals who are lost to follow-up pre-treatment. This potential needs rigorous evaluation.

TB patient cost surveys provide important information on how health service delivery and financing can be changed towards patient-centred care to eliminate TB patient costs, as well as enhancing social protection measures. ${ }^{3}$ They are a powerful tool, allowing us to focus on solving the financial plight and eliminating the barriers for presumptive and TB patients through collaboration with relevant stakeholders within and across the health sector. For example, addressing the problem of pre-diagnosis medical costs involves streamlining the TB patient pathway, expanding access to rapid molecular testing and digital X-rays, intensifying contact investigation and case finding, ${ }^{5}$ looking for synergies with programmes on HIV, nutrition and diabetes.

Ghana, the first country in Africa to conduct a national TB patient cost survey, ${ }^{6}$ recently developed a national roadmap to eliminate financial catastrophe for TB patients through a broad multisectoral agenda. The first policy action of this roadmap has led to the decision of the Ghana National Health Service and National Health Insurance Authority to enrol all TB patients in the National Health Insurance Schemefree of charge. This should serve as an example and reminder to other countries and technical partners that ongoing efforts to measure TB-related costs can and should promote bold action to eliminate the catastrophic costs and suffering due to TB, and ultimately lead to TB elimination.

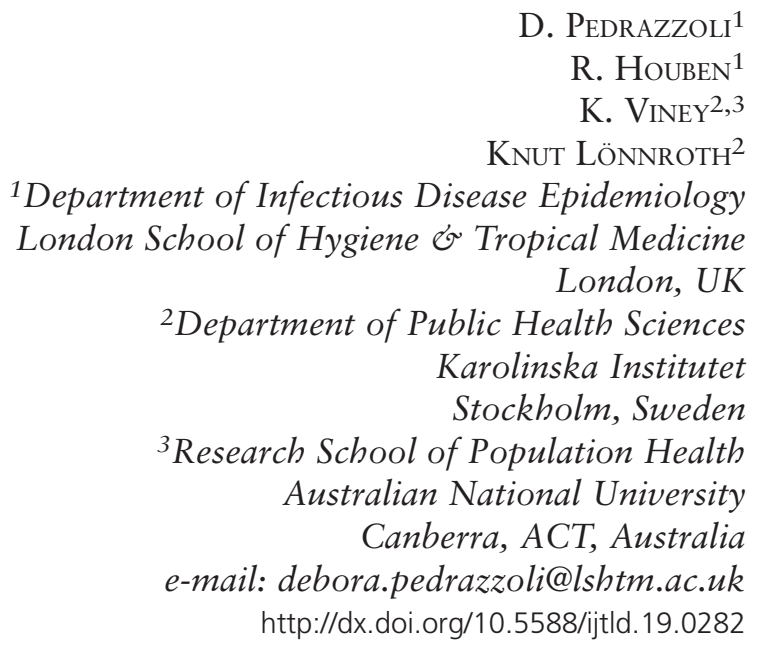

Conflicts of interest: none declared.

\section{References}

1 Timire C, Sandy C, Harries A D. Eliminating tuberculosis by 2035: tackling the financial barriers at all stages of the cascade of care. Int J Tuberc Lung Dis 2019; 23: 1235.

2 Rachow A, Ivanova O, Wallis R, et al. TB sequel: incidence, pathogenesis and risk factors of long-term medical and social sequelae of pulmonary TB: a study protocol. BMC Pulm Med 2019; 19(1): 4 .

3 World Health Organization. Tuberculosis patient cost surveys: a handbook. Geneva, Switzerland: WHO, 2017.

4 Health and Social Protection Action Research \& Knowledge Sharing (SPARKS) Network. Rationale, objectives and work plan. Report from the first SPARKS consultation, 15-16 December 2016. Stockholm, Sweden: SPARKS, 2017.

5 Morishita F, Yadav R P, Eang M T, Saint S, Nishikiori N. Mitigating financial burden of tuberculosis through active case finding targeting household and neighbourhood contacts in Cambodia. PLoS One 2016; 11(9): e0162796.

6 Pedrazzoli D, Siroka A, Boccia D, et al. How affordable is TB care? Findings from a nationwide TB patient cost survey in Ghana. Trop Med Int Health 2018; 23(8): 870-878. 